\title{
Quantized Conductance of Point Contacts in a Two-Dimensional Electron Gas
}

\author{
B J van Wees \\ Department of Applied Physics Delft Unuversity of Technology 2628 CJ Delft The Netherlands \\ H van Houten, C W J Beenakker, and J G Willamson, \\ Philips Research Laboratories 5600 JA Eindhoven, The Netherlands \\ L P Kouwenhoven and D van der Marel \\ Department of Applied Physics Delft Unitersity of Technology $2628 \mathrm{CJ}$ Delft, The Netherlands \\ and \\ C $\mathrm{T}$ Foxon \\ Philips Research Laboratories Redhill, Surrey RHI 5HA United Kingdom \\ (Recelved 31 December 1987)
}

\begin{abstract}
Ballistic point contacts, defined in the two-dimensional electron gas of a GaAs-AlGaAs heterostructure, have been studied in zero magnetic field The conductance changes in quantized steps of $e^{2} / \pi \hbar$ when the width, controlled by a gate on top of the heterojunction, is varied Up to sixteen steps are observed when the point contact is widened from 0 to $360 \mathrm{~nm}$ An explanation is proposed, which assumes quantized transverse momentum in the point-contact regıon

PACS numbers $7220 \mathrm{Jv} 7340 \mathrm{Cg} 7340 \mathrm{Lq}$
\end{abstract}

As a result of the high mobility dttainable in the twodımensional electron gas (2DEG) in GaAs-AlGaAs heterostructures it is now becoming feasible to study ballistic transport in small devices ${ }^{1-6}$ In metals ideal tools for such studies are constrictions having a width $W$ and length $L$ much smaller than the mean free path $l_{e}$ These are known as Sharvin point contacts ${ }^{7}$ Because of the ballistic transport through these constrictions, the resistance is determined by the point-contact geometry only Point contacts have been used extensively for the study of elastic and inelastic electron scattering With use of brased point contacts, electrons can be injected into metals at energies above the Fermi level This allows the study of the energy dependence of the scattering mechanisms ${ }^{8} W_{1}$ th the use of a geometry containing two point contacts, with separation smaller than $l_{e}$, electrons injected by a point contact can be focused into the other contact, by the application of a magnetic field This technique (transverse electron focusing) has been applied to the detalled study of Fermı surfaces ${ }^{9}$

In this Letter we report the first experimental study of the resistance of ballistic point contacts in the 2DEG of high-mobility GaAs-AlGaAs heterostructures The single-point contacts discussed in this paper are part of a double-point-contact device The results of transverse electron focusing in these devices will be published elsewhere ${ }^{10}$ The point contacts are defined by electrostatic depletion of the 2DEG underneath a gate This method, which has been used by several authors for the study of 1D conduction, ${ }^{12}$ offers the possibility to control the width of the point contact by the gate voltage Control of the width is not feasible in metal point contacts
The classical expression for the conductance of a point contact in two dimensions (see below) is

$$
G=\left(e^{2} / \pi \hbar\right) k_{\mathrm{F}} W / \pi
$$

in which $k_{\mathrm{F}}$ is the Fermı wave vector and $W$ is the width of the contact This expression is valid if $l_{e} \gg W$ and the Fermi wavelength $\lambda_{\mathrm{F}} \ll W$ The first condition is satisfied in our devices, which have a maximum width $W_{\max }$ $\approx 250 \mathrm{~nm}$ and $l_{e}=85 \mu \mathrm{m}$ The second condition should also hold when the devices have the maximum width We expect quantum effects to become important when the width becomes comparable to $\lambda_{\mathrm{F}}$, which is $42 \mathrm{~nm}$ in our devices In this way we are able to study the transition from classical to quantum ballistic transport through the point contact

The point contacts are made on high-mobility molecular-beam-epitaxy-grown GaAs-AlGaAs heterostructures The electron density of the material is $356 \times 10^{15} / \mathrm{m}^{2}$ and the mobility $85 \mathrm{~m}^{2} / \mathrm{V} \mathrm{s}$ (at $06 \mathrm{~K}$ ) These values are obtained from the devices containing the studied point contacts A standard Hall bar geometry is defined by wet etching Using electron-beam lithography, a metal gate is made on top of the heterostructure, with an opening $250 \mathrm{~nm}$ wide (inset in Fig 1) The point contacts are defined by the application of a negatıve voltage to the gate At $V_{g}=-06 \mathrm{~V}$ the electron gas underneath the gate is depleted, the conduction taking place through the point contact only At this voltage the point contacts have their maximum width $W_{\max }$, about equal to the opening between the gates By a further decrease of the gate voltage, the width of the point contacts can gradually be reduced, untıl they are fully 


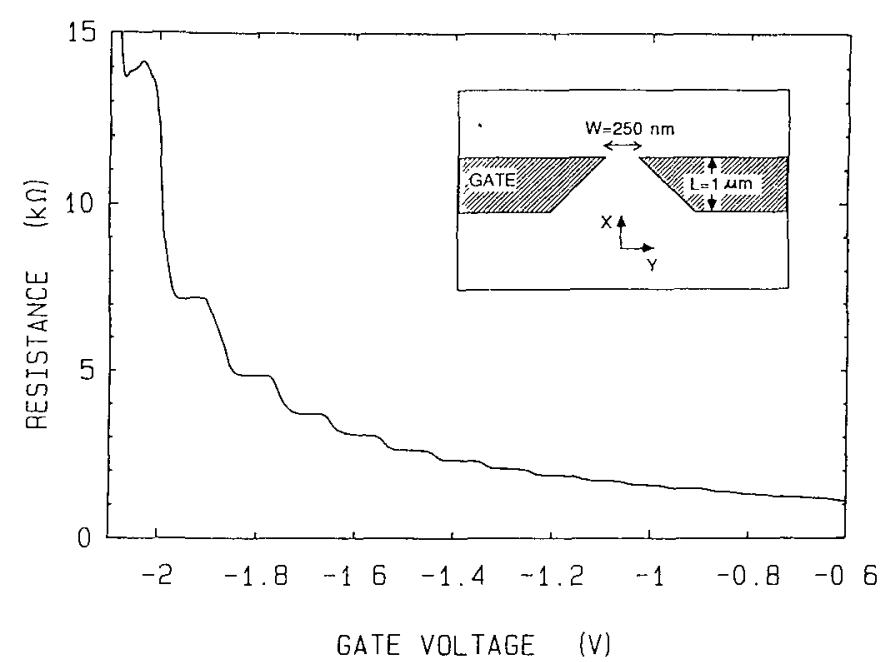

FIG. 1. Point-contact resistance as a function of gate voltage at $0.6 \mathrm{~K}$. Inset: Point-contact layout.

pinched off at $V_{g}=-2.2 \mathrm{~V}$.

We measured the resistance of several point contacts as a function of gate voltage. The measurements were performed in zero magnetic field, at $0.6 \mathrm{~K}$. An ac lockin technique was used, with voltages across the sample kept below $k T / e$, to prevent electron heating. In Fig. 1 the measured resistance of a point contact as a function of gate voltage is shown. Unexpectedly, plateaus are found in the resistance. In total, sixteen plateaus are observed when the gate voltage is varied from -0.6 to $-2.2 \mathrm{~V}$. The measured resistance consists of the resistance of the point contact, which changes with gate voltage, and a constant series resistance from the $2 \mathrm{DEG}$ leads to the point contact. As demonstrated in Fig. 2, a plot of the conductance, calculated from the measured resistance after subtraction of a lead resistance of $400 \Omega$, shows clear plateaus at integer multiples of $e^{2} / \pi \hbar$. The above value for the lead resistance is consistent with an estimated value based on the lead geometry and the resistivity of the 2DEG. We do not know how accurate the quantization is. In this experiment the deviations from integer multiples of $e^{2} / \pi \hbar$ might be caused by the uncertainty in the resistance of the $2 D E G$ leads. Inserting the point-contact resistance at $V_{g}=-0.6 \mathrm{~V}(750 \Omega)$ into Eq. (1) we find for the width $W_{\max }=360 \mathrm{~nm}$, in reason-

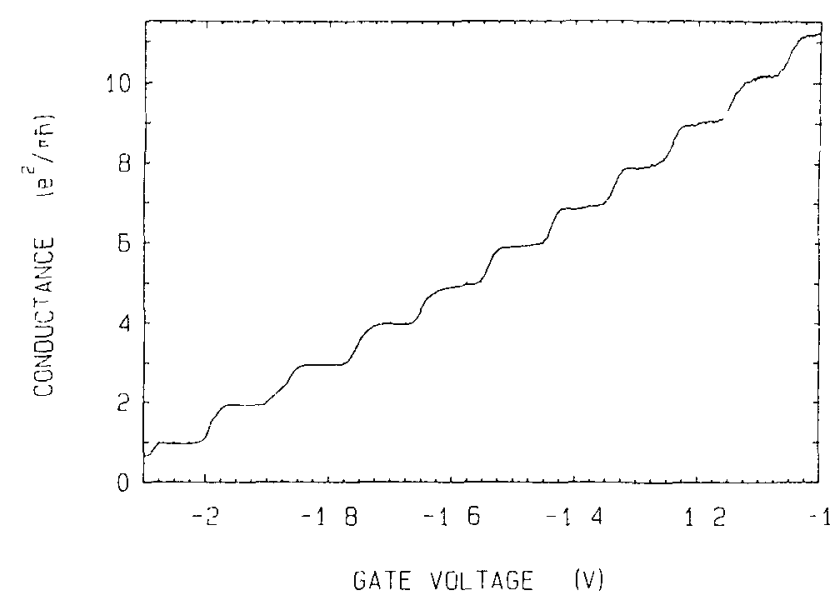

FIG. 2. Point-contact conductance as a function of gate voltage, obtained from the data of Fig. 1 after subtraction of the lead resistance. The conductance shows plateaus at multiples of $e^{2} / \pi \hbar$.

able agreement with the lithographically defined width between the gate electrodes.

The average conductance increases almost linearly with gate voltage. This indicates that the relation between the width and the gate voltage is also almost linear. From the maximum width $W_{\max }(360 \mathrm{~nm})$ and the total number of observed steps (16) we estimate the increase in width between two consecutive steps to be 22 nm.

We propose an explanation of the observed quantization of the conductance, based on the assumption of quantized transverse momentum in the contact constriction. In principle this assumption requires a constriction much longer than wide, but presumably the quantization is conserved in the short and narrow constriction of the experiment. The point-contact conductance $G$ for ballistic transport is given by ${ }^{7,11}$

$$
G=e^{2} N_{0} W(\hbar / 2 m)\left\langle\left|k_{x}\right|\right\rangle .
$$

The brackets denote an average of the longitudinal wave vector $k_{x}$ over directions on the Fermi circle, $N_{0}$ $=m / \pi \hbar^{2}$ is the density of states in the two-dimensional electron gas, and $W$ is the width of the constriction. The Fermi-circle average is taken over discrete transverse wave vectors $k_{y}= \pm n \pi / W(n=1,2, \ldots)$, so that we can write

$$
\left\langle\left|k_{x}\right|\right\rangle=\frac{1}{2 \pi k_{\mathrm{F}}} \int d^{2} k\left|k_{x}\right| \delta\left(k-k_{\mathrm{F}}\right) \frac{2 \pi}{W} \sum_{n=1}^{\infty} \delta\left(k_{y}-\frac{n \pi}{W}\right) .
$$

Carrying out the integration and substituting into Eq. (2), one obtains the result

$$
G=\sum_{n=1}^{N_{c}} \frac{e^{2}}{\pi \hbar}
$$

where the number of channels (or one-dimensional subbands) $N_{c}$ is the largest integer smaller than $k_{\mathrm{F}} W / \pi$. For 
$k_{\mathrm{F}} W \gg 1$ this expression reduces to the classical formula [Eq. (1)]. Equation (4) tells us that $G$ is quantized in units of $e^{2} / \pi \hbar$ in agreement with the experimental observation. With the increase of $W$ by an amount of $\lambda_{F} / 2$, an extra channel is added to the conductance. This compares well with the increase in width between two consecutive steps, determined from the experiment. Equation (4) may also be viewed as a special case of the multichannel Landauer formula, ${ }^{12-14}$

$$
G=\frac{e^{2}}{\pi \hbar} \sum_{n, m=1}^{N_{c}}\left|t_{n m}\right|^{2},
$$

for transmission coefficients $\left|t_{n m}\right|^{2}=\delta_{n m}$ corresponding to ballistic transport with no channel mixing.

It is interesting to note that this multichannel Landauer formula has been developed to describe the idealized case of the resistance of a quantum wire, connected to massive reservoirs, in which the inelastic-scattering events are thought to take place exclusively. As discussed by Imry, ${ }^{13}\left|t_{n m}\right|^{2}=\delta_{n m}$ corresponds to the case that elastic scattering is absent in the wire also. The fact that the conductance $G=N_{c} e^{2} / \pi \hbar$ of such an ideal wire is finite ${ }^{15}$ is a consequence of the inevitable contact resistances associated with the connection to the thermalizing reservoirs. The findings described in this Letter may imply that we have realized an experimental system which closely approximates the behavior of idealized mesocopic systems.

In summary we have reported the first measurements of the conductance of single ballistic point contacts in a two-dimensional electron gas. A novel quantum effect is found: The conductance is quantized in units of $e^{2} / \pi \hbar$.

We would like to thank J. M. Lagemaat, C. E. Timmering, and L. W. Lander for technological support and L. J. Geerligs for assistance with the experiments. We thank the Delft Center for Submicron Technology for the facilities offered and the Stichting voor Fundamenteel Onderzoek der Materie (FOM) for financial support.
${ }^{1}$ T. J. Thornton, M. Pepper, H. Ahmed, D. Andrews, and G. J. Davies, Phys. Rev. Lett. 56, 1198 (1986).

${ }^{2}$ H. Z. Zheng, H. P. Wei, D. C. Tsui, and G. Weimann, Phys. Rev. B 34, 5635 (1986).

${ }^{3}$ K. K. Choi, D. C. Tsui, and S. C. Palmateer, Phys. Rev. B 32, 5540 (1985).

${ }^{4}$ H. van Houten, C. W. J. Beenakker, B. J. van Wees, and J. E. Mooij, in Proceedings of the Seventh International Conference on the Physics of Two-Dimensional Systems, Santa Fe, 1987, Surf. Sci. (to be published).

${ }^{5}$ G. Timp, A. M. Chang, J. E. Cunningham, T. Y. Chang, P. Mankiewich, R. Behringer, and R. E. Howard, Phys. Rev. Lett. 58, 2814 (1987).

${ }^{6}$ G. Timp, A. M. Chang, P. Mankiewich, R. Behringer, J. E. Cunningham, T. Y. Chang, and R. E. Howard, Phys. Rev. Lett. 59, 732 (1987).

${ }^{7}$ Yu.V. Sharvin, Zh. Eksp. Teor. Fiz. 48, 984 (1965) [Sov. Phys. JETP 21, 655 (1965)].

${ }^{8}$ For a review, see I. K. Yanson and O. I. Shklyarevskii, Fiz. Nizk. Temp. 12, 899 (1986) ISov. J. Low Temp. Phys. 12, 509 (1986)].

${ }^{9}$ P. C. van Son, H. van Kempen, and P. Wyder, Phys. Rev. Lett. 58, 1567 (1987).

${ }^{10} \mathrm{H}$. van Houten, B. J. van Wees, J. E. Mooij, C. W. J. Beenakker, J. G. Williamson, and C. T. Foxon (to be published).

${ }^{11}$ I. B. Levinson, E. V. Sukhorukov, and A. V. Khaetskii, Pis'ma Zh. Eksp. Teor. Fiz. 45, 384 (1987) [JETP Lett. 45, 488 (1987)].

${ }^{12}$ R. Landauer, IBM J. Res. Dev, 1, 223 (1957); R. Landauer, Phys. Lett. 85A, 91 (1981).

${ }^{13}$ M. Buttiker, Y. Imry, R. Landauer, and S. Pinhas, Phys. Rev. B 31, 6207 (1985). For a survey, see Y. Imry, in Directions in Condensed Matter Physics, edited by G. Grinstein and G. Mazenko (World Scientific, Singapore, 1986), Vol. 1, p. 102 .

${ }^{14}$ D. S. Fisher and P. A. Lee, Phys. Rev. B 23, 6851 (1981).

${ }^{15}$ The original Landauer formula (Ref. 12) containing the ratio of transmission and reflection coefficients does give an infinite conductance for a perfect system. However, this formula excludes contributions from the contact resistances. 